\title{
Molecular analysis of ANT1, TWINKLE and POLG in patients with multiple deletions or depletion of mitochondrial DNA by a dHPLC-based assay
}

\author{
Mourad Naïmi ${ }^{1,2,14}$, Sylvie Bannwarth ${ }^{1,14}$, Vincent Procaccio ${ }^{3}$, Jean Pouget ${ }^{4}$, \\ Claude Desnuelle ${ }^{5}$, Jean-François Pellissier ${ }^{6}$, Agnes Rötig ${ }^{7}$, Arnold Munnich ${ }^{7}$, \\ Patrick Calvas ${ }^{8}$, Christian Richelme ${ }^{9}$, Philippe Jonveaux ${ }^{10}$, Giovanni Castelnovo ${ }^{11}$, \\ Melvin Simon ${ }^{3}$, Michel Clanet ${ }^{12}$, Douglas Wallace ${ }^{3}$ and Véronique Paquis-Flucklinger ${ }^{\star}, 1,13$
}

\footnotetext{
${ }^{1}$ Department of Medical Genetics, Archet 2 Hospital, CHU Nice, France; ${ }^{2}$ INSERM U145, Medicine School, Nice, France; ${ }^{3}$ Center for Molecular and Mitochondrial Medicine and Genetics, University of California, Irvine, USA; ${ }^{4}$ Department of Neurology, Timone Hospital, CHU Marseille, France; ${ }^{5}$ Department of Reeducation, Archet 2 Hospital, CHU Nice, France; ${ }^{6}$ Department of Neuropathology, Timone Hospital, CHU Marseille, France; ${ }^{7}$ INSERM U393, Necker Hospital, Paris, France; ${ }^{8}$ Department of Genetics, Purpan Hospital, CHU Toulouse, France; ${ }^{9}$ Department of Pediatrics, Archet 2 Hospital, CHU Nice, France; ${ }^{10}$ Department of Genetics, Brabois Hospital, CHU Nancy, France; ${ }^{11}$ Department of Neurology, Caremeau Hospital, CHU Nimes, France; ${ }^{12}$ Department of Neurology, Purpan Hospital, CHU Toulouse, France; ${ }^{13}$ UMR CNRS 6543, Medicine School, Nice, France
}

ANT1, TWINKLE and POLG genes affect mtDNA stability and are involved in autosomal dominant PEO, while mutations in POLG are responsible for numerous clinical presentations, including autosomal recessive PEO, sensory ataxic neuropathy, dysarthria and ophthalmoparesis (SANDO), spino-cerebellar ataxia and epilepsy (SCAE) or Alpers syndrome. In this study, we report on the mutational analysis of ANT1, TWINKLE and POLG genes in 15 unrelated patients, using a dHPLC-based protocol. This series of patients illustrates the large array of clinical presentations associated with mtDNA stability defects, ranging from isolated benign PEO to fatal Alpers syndrome. A total of seven different mutations were identified in six of 15 patients (40\%). Six different recessive mutations were found in POLG, one in TWINKLE while no mutation was identified in ANT1. Among the POLG mutations, three are novel and include two missense and one frameshift changes. Seventeen neutral changes and polymorphisms were also identified, including four novel neutral polymorphisms. Overall, this study illustrates the variability of phenotypes associated with mtDNA stability defects, increases the mutational spectrum of POLG variants and provides an efficient and reliable detection protocol for ANT1, TWINKLE and POLG mutational screening.

European Journal of Human Genetics (2006) 14, 917-922. doi:10.1038/sj.ejhg.5201627; published online 26 April 2006

Keywords: mtDNA multiple deletions; mtDNA depletion; dHPLC; ANT1; TWINKLE; POLG

\footnotetext{
*Correspondence: Professor V Paquis-Flucklinger, Department of Medical Genetics, Archet 2 Hospital, 151, Route de Saint Antoine de Ginestière, BP 3079, 06202 Nice cedex 3, France.

Tel: + 334920364 60; Fax : + 334920364 65;

E-mail: paquis@hermes.unice.fr

${ }^{14}$ These authors contributed equally to the study.

Received 31 October 2005; revised 21 February 2006; accepted 7 March 2006; published online 26 April 2006
}

\section{Introduction}

Adenine nucleotide translocator 1 (ANT1; OMIM \#103220), mitochondrial replicative helicase Twinkle (OMIM \#606075) and polymerase gamma (POLG; OMIM \#174763) genes affect mtDNA stability, and their mutations cause autosomal dominant progressive external ophthalmoplegia (adPEO). ${ }^{1-3}$ POLG mutations have also been found in autosomal recessive PEO (arPEO) and it is thought that 
Table 1 Clinical features of patients

\begin{tabular}{|c|c|c|c|c|c|c|c|}
\hline Patient & Sex & $\begin{array}{l}\text { Age } \\
\text { (years) }\end{array}$ & $\begin{array}{l}\text { Onset } \\
\text { (years) }\end{array}$ & $\begin{array}{l}\text { Family } \\
\text { history }\end{array}$ & $\begin{array}{l}\text { Ptosis/ } \\
\text { PEO }\end{array}$ & Other symptoms & MtDNA alterations \\
\hline $\begin{array}{l}\mathrm{PA}^{+} \\
\mathrm{PB}^{\dagger} \\
\mathrm{PC}^{-}\end{array}$ & $\begin{array}{l}F \\
M \\
M\end{array}$ & $\begin{array}{l}63 \\
15 \\
63\end{array}$ & $\begin{array}{r}50 \\
8 \\
40\end{array}$ & $\begin{array}{l}\text { Sporadic } \\
\text { Sporadic } \\
\text { Sporadic }\end{array}$ & $\begin{array}{l}+ \\
+ \\
+\end{array}$ & $\begin{array}{l}\text { None } \\
\text { SANDO } \\
\text { Weakness of lower limbs, ataxia, cognitive } \\
\text { impairment }\end{array}$ & $\begin{array}{l}\text { Multiple deletions in muscle } \\
\text { // } \\
\text { // }\end{array}$ \\
\hline PD & M & 57 & 56 & Sporadic & + & $\begin{array}{l}\text { Exercise intolerance, dysphagia, diabetes, } \\
\text { sensory axonal neuropathy }\end{array}$ & $/ /$ \\
\hline $\mathbf{P E}^{\dagger}$ & M & 4 & 3 & Recessive & - & Alpers syndrome & Depletion in liver \\
\hline $\mathrm{PF}$ & M & 82 & 78 & Sporadic & + & $\begin{array}{l}\text { Myopathy, dysphagia, dysphonia, ataxia, } \\
\text { tremor, hypoacousia, diabetes }\end{array}$ & Multiple deletions in muscle \\
\hline PG & $\mathbf{F}$ & 45 & 18 & Dominant & + & Myopathy, dysphagia, dysphonia, dyspnea & // \\
\hline $\mathrm{PH}$ & $\mathrm{F}$ & 52 & $\mathrm{Ch}$ & Recessive & - & Myopathy & // \\
\hline $\mathrm{PI}$ & $\mathrm{M}$ & 62 & 58 & Dominant & - & Myopathy, ataxia, frontal dementia & // \\
\hline PJ & $\mathrm{F}$ & 63 & 54 & Dominant & - & $\begin{array}{l}\text { Optic atrophy, myopathy, ataxia, sensory } \\
\text { axonal neuropathy }\end{array}$ & // \\
\hline PK & M & 54 & 18 & Sporadic & + & Cardiomyopathy, myopathy, strokes, epilepsy & // \\
\hline PL & $\mathbf{F}$ & 27 & 20 & Sporadic & + & SCAE & // \\
\hline PM & $\mathrm{F}$ & 30 & 5 & Sporadic & - & $\begin{array}{l}\text { Epilepsy, bilateral deafness, sensory-motor } \\
\text { axonal neuropathy }\end{array}$ & // \\
\hline $\mathrm{PN}^{\dagger}$ & $\mathrm{F}$ & $\mathrm{Nb}$ & $\mathrm{Nb}$ & Sporadic & - & Hepatocerebral failure & Depletion in liver \\
\hline $\mathrm{PO}^{\dagger}$ & $\mathbf{F}$ & $15 \mathrm{~m}$ & $7 \mathrm{~m}$ & Sporadic & - & Alpers syndrome & $/ /$ \\
\hline
\end{tabular}

PEO: progressive external ophthalmoplegia; SANDO: sensory ataxic neuropathy dysarthria ophthalmoplegia syndrome; SCAE: spinocerebellar ataxia epilepsy syndrome; ${ }^{\dagger}$ : Died; m: months; Ch: childhood; Nb: newborn; $(+)$ : present; $(-)$ : absent; //: same as upper. Patients for whom the disease causing mutations were identified are in bold.

mutations in this gene could explain $45 \%$ of sporadic or familial PEO cases with multiple mtDNA deletions. ${ }^{4}$ Additional clinical presentations comprising mtDNA deletions have been described recently. They include autosomal recessive sensory ataxic neuropathy with dysarthria and ophthalmoplegia (SANDO), which is associated with mutations in either POLG $G^{5}$ or TWINKLE $E^{6}$ genes, parkinsonism with premature menopause, mitochondrial recessive ataxia syndrome (MIRAS), and juvenile spino-cerebellar ataxia-epilepsy syndrome (SCAE), all associated with POLG mutations. ${ }^{7,8}$ Furthermore, mutations in POLG have also been associated with mtDNA depletion in patients affected by Alpers syndrome or by early hepatocerebral failure. ${ }^{9-11}$ In order to improve the molecular diagnosis in such patients, we have developed a new dHPLC assay for the complete screening of ANT1, TWINKLE and POLG coding regions and exon-intron boundaries. We report here the results of this screening in a series of 15 patients, illustrating the heterogeneity of phenotypes associated with mtDNA instability. All selected patients carried either multiple mtDNA deletions in muscle or mtDNA depletion in liver (Table 1).

\section{Materials and methods Subjects}

The clinical findings and family histories are summarized in Table 1 . The pedigrees are given in Figure 1.

\section{DNA extraction and PCR conditions}

Blood samples were obtained from patients and all available family members after informed consent was given. Genomic DNA was extracted from leukocytes by standard procedure. POLG, ANT1 and TWINKLE genes were PCR amplified with primers listed in Supplementary Tables 1, 2 and 3. Different Taq DNA polymerases were used in order to obtain dHPLC best results for each amplified exon (Supplementary Table 4).

\section{Cloning of wild-type DNA fragments}

To detect homozygous variants, we generated wild-type DNA fragments. Each PCR product of ANT1, TWINKLE and $P O L G$ genes, resulting from control individuals, were subcloned into pGEM-T Easy Vector (Promega, Charbonnieres-Les-Bains, France). All wild-type clones were sequenced and then, used as controls in dHPLC.

\section{dHPLC analysis}

The dHPLC system used in this study is a Transgenomic Wave Nucleic Acid Fragment Analysis System (Transgenomic $^{\mathrm{TM}}$, Crewe, UK). Optimal buffer gradients and mobile phase temperatures, which were determined by the Transgenomic software (Navigator ${ }^{\mathrm{TM}}$ software) are indicated in Supplementary Tables 1, 2 and 3. For homozygous variant detection, PCR products amplified from patients were combined with equal amounts of PCR products amplified from control DNA.

\section{Sequencing analysis}

PCR fragments were purified on Montage PCR columns (Millipore SA, SaintQuentin, France) and sequenced on a ABI 310 automated sequencer with D-Rhodamine cycle 


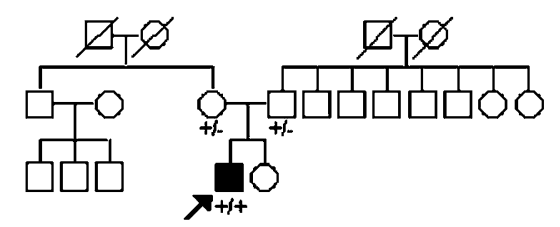

Family B

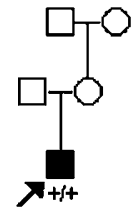

Family C

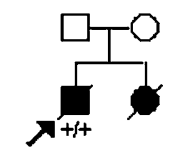

Family E

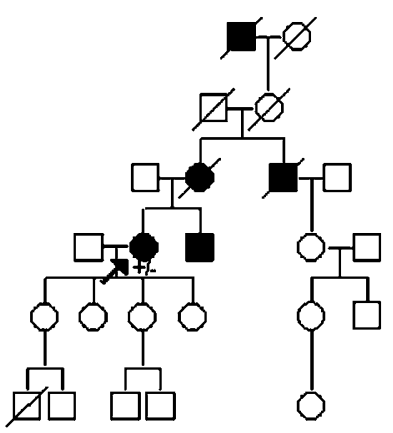

Family G

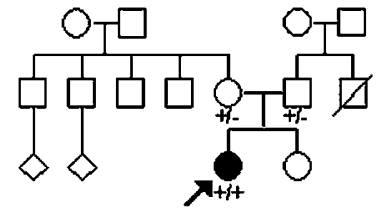

Family L

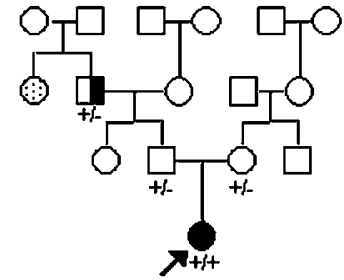

Family 0

\section{Affected individual}

Mental retardation and epilepsy

Muscle weakness without PEO

Figure 1 Pedigrees of six patients with POLG or TWINKLE mutations. +: presence of POLG mutation; -: absence of POLG mutation (families B, C, $\mathrm{E}, \mathrm{L}, \mathrm{O}) ;+$ : presence of TWINKLE mutation; -: absence of TWINKLE mutation (family G).

Table 2A Mutations detected in the study

\begin{tabular}{lllll}
\hline Patient & Gene & Nucleotide change & Amino-acid change & Type of mutation \\
\hline B & POLG & {$[$ c.911T $>$ G]* } & [L304R]* & Missense \\
C & POLG & c.1139G $>$ A $;$ c1399G $>$ A & G380D; A467T & Missense; Missense \\
E & POLG & c.975_976insC; $1399 \mathrm{G}>$ A & T326fsX387; A467T & Frameshift/Truncation; Missense \\
G & TWINKLE & c.1121G $>$ A & R374Q & Missense \\
L & POLG & c.911T $>$ G; c.2243G $>$ C & L304R; W748S & Missense; Missense \\
O & POLG & c.2740A $>$ C c c1399G $>$ A & T914P; A467T & Missense; Missense \\
\hline
\end{tabular}

Homozygous variations are indicated with an asterisk.

New sequence variations are in bold face.

sequencing ready reaction kit (PE Applied Biosystems, Foster City, CA).

\section{Results and discussion}

Our principal aims were to determine the ANT1, TWINKLE and POLG mutational spectrum in a series of patients, and to develop an efficient molecular test for mutational analysis of genes involved in mtDNA stability. The entire ANT1, TWINKLE and POLG coding regions and exonintron boundaries were screened by dHPLC in patients with neuromuscular symptoms. POLG only was analyzed in the three children deceased from hepatocerebral failure. All fragments giving an abnormal dHPLC profile were further analyzed by direct sequencing and a total of seven different probable pathogenic alleles were identified in six patients (Table 2A). Some of the dHPLC elution profiles are presented in Figure 2. Interestingly, the detection of A467T mutation in exon 7 of $P O L G$ was seen with one temperature only (Figure 2c). Several additional polymorphisms were also identified (Table 2B). In order to evaluate the reliability of our method, all fragments giving a normal dHPLC profile were also systematically sequenced and no discrepancy was observed between sequencing and dHPLC analysis.

Five patients out of 15 (33\%) had POLG recessive mutations. POLG contains two domains, a DNA polymerase and a $3^{\prime}-5^{\prime}$ exonuclease domain separated by a conserved inter-domain region known as spacer region. ${ }^{12,13}$ 

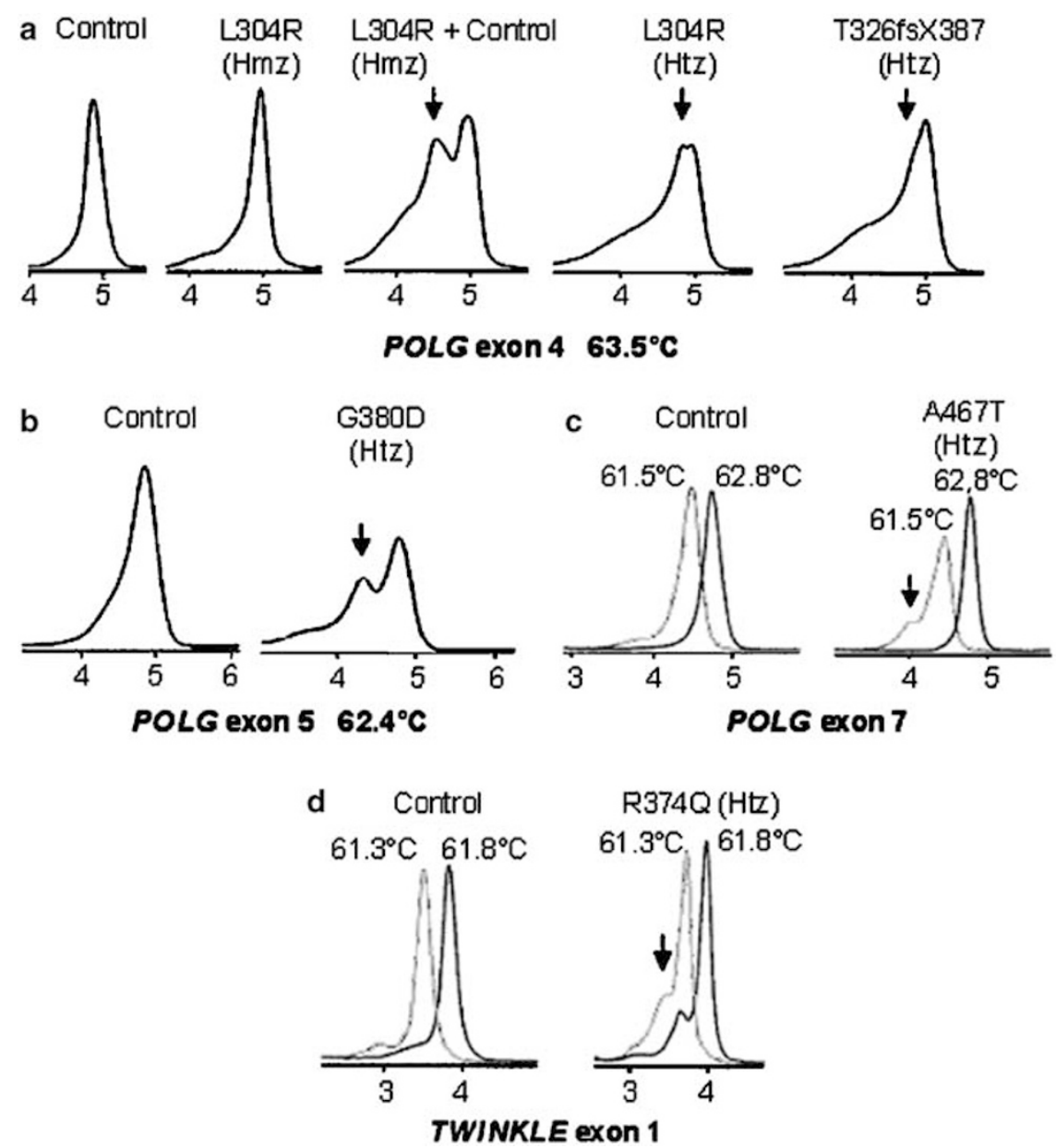

Figure 2 dHPLC elution profiles of four POLG and one TWINKLE mutations. Patient status is indicated above elution profiles. DNA-heteroduplexes are indicated by an arrow. Amplicons and oven temperature are indicated. Hmz: Homozygous; Htz: Heterozygous. (a) Elution profiles of POLG exon 4 amplicons. From left to right: amplicon obtained from a control; amplicon from patient $B$, alone and mixed with a wild-type amplicon; amplicons from patient $L$ and $E$, respectively. (b) Elution profiles of POLG exon 5 amplicons. Amplicon from a control (left) and patient C (right). (c) Elution profiles of POLG exon 7 amplicon. Amplicon from a control (left) and patient $\mathrm{E}$ (right) at $61.5^{\circ} \mathrm{C}$ (gray) and $62.8^{\circ} \mathrm{C}$ (black). (d) Elution profiles of $T W I N K L E$ exon 1. Amplicon from a control (left) and patient $\mathrm{G}$ (right) at $61.3^{\circ} \mathrm{C}$ (gray) and $61.8^{\circ} \mathrm{C}$ (black).

Patient B, who presented with a SANDO phenotype, resulted to be homozygous for the c.911T $>\mathrm{G}$ mutation leading to a L304R amino-acid change (Table 2A). This highly conserved amino acid is located between exonuclease domains II and III of POLG. This mutation has already been reported in arPEO Belgian families presenting with features of the SANDO syndrome, but only in combination with the c.1399G $>$ A substitution (A467T). ${ }^{5}$ We describe, for the first time, the L304R mutation in a homozygous state in a patient presenting with a SANDO phenotype.

The A467T mutation was found in a heterozygous state in patient $\mathrm{C}$, in combination with a new c.1139G $>\mathrm{A}$ substitution. This new mutation leads to a G380A aminoacid change affecting a highly conserved residue lying between the exonuclease motifs ExoII and ExoIII. This patient, who presented with a PEO phenotype, was a sporadic case and the pathogenicity of this new mutation was impossible to be assessed by segregation analysis. Nevertheless, we did not find the c.1139G > A substitution (G380D) in 300 healthy french control chromosomes.The A467T substitution was also found in patient $\mathrm{E}$, in combination with a new c.975_976insC mutation, which leads to a premature termination codon (T326fsX387). The A467T mutation, which maps within the conserved $\gamma 1$ element of POLG spacer, has already been described in Alpers disease in a homozygous state or in combination with other mutations. ${ }^{10,11}$ Another patient, who presented with an Alpers syndrome (patient $\mathrm{O}$ ) also carried the A467T mutation in combination with a new c.1399G $>$ A substitution, leading to a highly conserved T914P amino-acid change, between DNA polymerase domains A and B. The first mutation was inherited from the father, while the second was inherited from the mother. Recently, it has 
Table 2B Polymorphisms and variants detected in the study

\begin{tabular}{|c|c|c|c|c|}
\hline Location & Nucleotide change & Amino-acid change & Patient & NCBI SNP ID \\
\hline \multicolumn{5}{|l|}{ POLG } \\
\hline \multirow[t]{2}{*}{ Exon 2} & c.156_157insCAG & E- & $K$ & \\
\hline & c. $159 \mathrm{~A}>\mathrm{G}$ & Q53Q & $B^{*}$ & \\
\hline Intron 2 & c. $660-46 \mathrm{G}>\mathrm{A}$ & $-\infty$ & $\mathrm{F}$ & rs3176170 \\
\hline Exon 5 & c. $1126 \mathrm{C}>\mathrm{T}$ & L376L & J & \\
\hline Intron 6 & c. $1250-43 \mathrm{C}>\mathrm{T}$ & - & j & rs2307444 \\
\hline Exon 10 & c. $1780 \mathrm{C}>\mathrm{T}$ & L594L & $\mathrm{K}$ & \\
\hline Intron 11 & c. $2071-22 \mathrm{~T}>\mathrm{C}$ & - & $B^{*}, E, F, J^{*}, K^{*}$ & rs2072267 \\
\hline Intron 12 & c. $2157+92 \mathrm{~T}>\mathrm{C}$ & - & $B^{*}, D, E, I, J^{*}, K$ & rs 2072266 \\
\hline Exon 13 & c. $2254 \mathrm{C}>\mathrm{T}$ & L752L & I & \\
\hline Intron 17 & c. $2734+39$ 40insGTAG & - & $B^{*}, D^{*}, E, F, I, J^{*}, K^{*}$ & rs2307433 \\
\hline \multirow[t]{2}{*}{ Intron 19} & c. $3105-11 \mathrm{~T}>\mathrm{C}$ & - & $\mathrm{D}, \mathrm{I}, \mathrm{J}^{*}, \mathrm{~K}$ & rs 2302084 \\
\hline & c. $3105-36 \mathrm{~A}>\mathrm{G}$ & - & $B^{*}, D, I, J, K$ & rs2246900 \\
\hline Exon 21 & c. $3428 \mathrm{~A}>\mathrm{G}$ & E1143G & $\mathrm{L}$ & \\
\hline Intron 21 & c. $3483-19 \mathrm{~T}>\mathrm{G}$ & - & $\mathrm{D}, \mathrm{I}, \mathrm{l}, \mathrm{K}$ & rs2307438 \\
\hline Exon 23 & c. $3708 \mathrm{G}>\mathrm{T}$ & Q1236H & J & rs3087374 \\
\hline $3^{\prime}$ UTR & c.3769_3770insG & - & $B^{*}, D, E, I^{*}, J, K$ & rs3087377 \\
\hline \multicolumn{5}{|l|}{ TWINKLE } \\
\hline Exon 1 & c. $1102 \mathrm{G}>\mathrm{A}$ & V3681 & K & rs17113613 \\
\hline
\end{tabular}

POLG and TWINKLE polymorphisms and sequence variants detected in patients.

Novel variants appear in bold type.

Patients who carry the variant in a homozygous state are marked with an asterisk.

The references of variants already described are indicated. Single nucleotide polymorphism (SNP) database: http://www.ncbi.nlm.nih.gov/entrez/ query.fcgi?db $=$ snp.

been shown that a single allele with the A467T substitution may manifest in a dominant manner, leading to late-onset ptosis. ${ }^{14}$ In our study, pedigree $\mathrm{O}$ analysis is consistent with this observation since the paternal grandfather, who is heterozygous for A467T, presented with muscular weakness but without PEO symptomatology (Figure 1). Finally, patient $\mathrm{L}$ was found to be a compound heterozygous carrying the c.911T $>\mathrm{G}$ (L304R) mutation in combination with the recently described c.2243G $>C$ substitution (W748S). Our patient carried the W748S mutation in cis with a c.3428A $>$ G (E1143G) variant. The L304R mutation was inherited from her mother while the W748S mutation, associated with E1143G, was inherited from her father. The W748S mutation is located in the conserved $\gamma 4$ region of the POLG spacer, has been associated with SCAE, Alpers and infantile hepatocerebral syndromes, ${ }^{7,9,10,15}$ and has always been described in combination with the E1143G variant in cis. From a practical point of view, the case of this young woman illustrates the interest of molecular analysis in mitochondrial diseases. She was pregnant and the identification of two POLG mutations in trans allowed us to give a reassuring genetic counselling.

One patient harbored a TWINKLE mutation which has already been described. ${ }^{1}$ Patient $\mathrm{G}$ was found to carry a c.1121G $>$ A transition, predicting a highly conserved R374Q amino-acid change of the TWINKLE protein sequence. R374Q was not found in the patient's daughter, the only healthy individual available in the pedigree. In our study, the R374Q mutation is associated with severe respiratory failure due to respiratory muscle weakness (pedigree G). No other TWINKLE or ANT1 mutations were found in the remaining pedigrees.

Overall, this study, which illustrates the variability of phenotypes associated with mtDNA stability defects, increases the mutational spectrum of $P O L G$ variants and provides an efficient and reliable detection protocol for ANT1, TWINKLE and POLG mutational screening.

\section{Acknowledgements}

This work was made possible by grants from the Centre Hospitalier Universitaire (CHU) de Nice. We thank Alice Blombou, Bernadette Chafino and Sabine Charabot for technical help.

\section{Conflicts of interest declared: none}

\section{Databases:}

ANT1 - OMIM: 103220; 609283 (PEOA2); GenBank: NT_022792. TWINKLE - OMIM: 606075; 609286 (PEOA3), 607459 (SANDO); GenBank: NT_030059, AF29004.

POLG - OMIM: 174763; 157640 (PEOA1), 258450 (PEOB), 607459 (SANDO), 203700 (PNDC), 251880 (MDS); GenBank: NT_033276, NM_002693.

Website: http://dir-apps.niehs.nih.gov/polg/index.cfm

\section{References}

1 Spelbrink JN, Li FY, Tiranti V et al: Human mitochondrial DNA deletions associated with mutations in the gene encoding Twinkle, a phage T7 gene 4-like protein localized in mitochondria. Nat Genet 2001; 28: 223-231.

2 Van Goethem G, Dermaut B, Lofgren A, Martin JJ, Van Broeckhoven C: Mutation of POLG is associated with progressive external ophthalmoplegia characterized by mtDNA deletions. Nat Genet 2001; 28: 211-212. 
3 Kaukonen J, Juselius JK, Tiranti V et al: Role of adenine nucleotide translocator 1 in mtDNA maintenance. Science 2000; 289: $782-785$.

4 Lamantea E, Tiranti V, Bordoni A et al: Mutations of mitochondrial DNA polymerase gammaA are a frequent cause of autosomal dominant or recessive progressive external ophthalmoplegia. Ann Neurol 2002; 52: 211-219.

5 Van Goethem G, Martin JJ, Dermaut B et al: Recessive POLG mutations presenting with sensory and ataxic neuropathy in compound heterozygote patients with progressive external ophthalmoplegia. Neuromuscul Disord 2003; 13: 133-142.

6 Hudson G, Deschauer M, Busse K, Zierz S, Chinnery PF: Sensory ataxic neuropathy due to a novel C10Orf2 mutation with probable germline mosaicism. Neurology 2005; 64: 371-373.

7 Winterthun S, Ferrari G, He L et al: Autosomal recessive mitochondrial ataxic syndrome due to mitochondrial polymerase gamma mutations. Neurology 2005; 64: 1204-1208.

8 Luoma P, Melberg A, Rinne JO et al: Parkinsonism, premature menopause, and mitochondrial DNA polymerase gamma mutations: clinical and molecular genetic study. Lancet 2004; 364: $875-882$.
9 Davidzon G, Mancuso M, Ferraris S et al: POLG mutations and Alpers syndrome. Ann Neurol 2005; 57: 921-923.

10 Ferrari G, Lamantea E, Donati A et al: Infantile hepatocerebral syndromes associated with mutations in the mitochondrial DNA polymerase-gammaA. Brain 2005; 128: 723-731.

11 Naviaux RK, Nguyen KV: POLG mutations associated with Alpers' syndrome and mitochondrial DNA depletion. Ann Neurol 2004; 55: 706-712.

12 Kaguni LS: DNA polymerase gamma, the mitochondrial replicase. Annu Rev Biochem 2004; 73: 293-320.

13 Luo N, Kaguni LS: Mutations in the spacer region of Drosophila mitochondrial DNA polymerase affect DNA binding, processivity, and the balance between Pol and Exo function. J Biol Chem 2005; 280: $2491-2497$.

14 Luoma PT, Luo N, Loscher WN et al: Functional defects due to spacer-region mutations of human mitochondrial DNA polymerase in a family with an ataxia-myopathy syndrome. Hum $\mathrm{Mol}$ Genet 2005; 14: 1907-1920.

15 Van Goethem G, Luoma P, Rantamaki M et al: POLG mutations in neurodegenerative disorders with ataxia but no muscle involvement. Neurology 2004; 63: 1251-1257.

Supplementary Information accompanies the paper on European Journal of Human Genetics website (http://www.nature.com/ejhg) 